O'Connor, R. and Laporte, C.Y., Using ISO/IEC 29110 to Harness Process Improvement in Very Small Entities, Workshop on SPI in SMEs, 18th European Software Process Improvement Conference, CCIS Vol. 172, Springer-Verlag, June 2011

\title{
Using ISO/IEC 29110 to Harness Process Improvement in Very Small Entities
}

\author{
Rory V. O'Connor ${ }^{1,2}$ Claude Y. Laporte ${ }^{3}$ \\ ${ }^{1}$ Lero, the Irish Software Engineering Research Centre, Ireland \\ ${ }^{2}$ Dublin City University, Dublin, Ireland \\ ${ }^{3}$ École de technologie supérieure, Montréal, Canada \\ Rory.OConnor@computing.dcu.ie,Claude.Y.Laporte@etsmtl.ca
}

\begin{abstract}
The recently published ISO/IEC 29110 standard Lifecycle profiles for Very Small Entities has at its core a Management and Engineering Guide [1] which are targeted at very small entities (enterprises, organizations, departments or projects) having up to 25 people [2], to assist them unlock the potential benefits of using standards which are specifically designed to address their needs. This paper will outline this new standard and the implementation of a series of pilot project initiative harnessing a set of detailed guidelines known as "Deployment Packages" to assist very small entities in understanding the potential usage of this new software process standard.
\end{abstract}

Keywords: VSE, ISO/IEC 29110, ISO, Standards

\section{Introduction}

For many small and very small software companies, implementing controls and structures to properly manage their software development activity is a major challenge. Administering software development in this way is usually achieved through the introduction of a software process. All software companies are not the same and vary according to factors including size, market sector, time in business, management style, product range and geographical location. For example, a software company operating in India may have a completely different set of operational problems to contend with to a software company in Israel or Ireland. Even within a single geographical area such as Ireland, the range of operational issues faced by a small local Irish-owned firm can be radically different to those affecting a multinational subsidiary. The fact that all companies are not the same, raises important questions for those who develop software process and process improvement models. To be widely adopted by the software industry, any process or process improvement model should be capable of handling the differences in the operational contexts of the companies making up that industry. But process improvement models, though highly publicized and marketed, are far from being extensively deployed and their influence in the software industry therefore remains more at a theoretical than practical level [3].

In a time when software quality is a key to competitive advantage, the use of ISO/IEC systems and software engineering standards remains limited to a few of the 
most popular ones. Research shows that small and very small companies can find it difficult to relate ISO/IEC standards to their business needs and to justify the application of the standards to their business practices [2, 3, 4]. Most of these companies don't have the expertise or can't afford the resources - in number of employees, cost, and time - or see a net benefit in establishing software life-cycle processes. There is sometimes a disconnect between the short-term vision of the company, looking at what will keep it in business for another six months or so, and the long-term or mid-term benefits of gradually improving the ways the company can manage its software development and maintenance. A primary reason cited by many small software companies for this lack of adoption of software engineering standards, is the perception that they have been developed for large software companies and not with the small organization in mind [3]. To date VSEs have no or very limited ways to be recognized, by large organizations, as enterprises that produce quality software systems within budget and calendar in their domain and may therefore be cut off from some economic activities.

Accordingly there is a need to help such organizations understand and use the concepts, processes and practices proposed in the ISO/IEC JTC1/SC7's international software engineering standards. The recently published ISO/IEC 29110 standard "Lifecycle profiles for Very Small Entities" [1] is aimed at addressing the issues identified above and addresses the specific needs of VSEs.

\section{Background}

This section will present a brief overview of the motivation behind the adoption of standards by software development organizations and discuss the particular issues faced by small and very small companies in the adoption of software process standards. It will also introduce the specific needs of one specific category of very small organization, known as a Very Small Entity (VSE).

\subsection{Standards and benefits}

Quality orientated process approaches and standards are maturing and gaining acceptance in many organizations. Standards emphasize communication and shared understanding more than anything. Examples are: any documentation is consistent and what is needed to meet the needs of the organization; all users understand the same meaning of words used - if one person says, 'Testing is completed 'all affected bodies understand what those words mean. This kind of understanding is not only important in a global development environment; even a small group working in the same office might have difficulties in communication and understanding of issues shared by all. Standards can help in these and other areas to make the business more profitable because less time is spent on non-productive work.

There are many potential benefits of using standards. From the a VSE perspective, the benefits that certification can provide include: increased competitiveness, greater customer confidence and satisfaction, greater software product quality, increased 
sponsorship for process improvement, decreased development risk, facilitation of marketing, and higher potential to export. While good internal software management might help meet the first five claims; the last two can only be the benefits of using a widely recognized standard.

Many Software Process Improvement (SPI) models have been developed to assist companies in this regard and purport to represent beacons of 'best practice'. Contained within the scope of these models, according to their supporters, lies the road to budgetary and schedule adherence, better product quality and improved customer satisfaction. Some large software organizations have used SPI 'best practice' models, such as the Capability Maturity Model Integration (CMMI) [5] and the ISO 9000 series [6]. More recently, agile methodologies have been used in SPI programmes as a way of improving delivery time and increasing customer satisfaction, and these agile approaches have been widely embraced by software organizations.

\subsection{Problems with standards}

Although commercial SPI models (such as CMMI) have been highly publicized and marketed, they are not being widely adopted and their influence in the software industry therefore remains more at a theoretical than practical level [4]. In the case of CMMI, evidence for this lack of adoption can be seen by examining the SEI (Software Engineering Institute) CMMI data for the three year period March 2008 to March 2011[7], which shows that worldwide during that period less than 3,500 individual appraisals were reported, which includes many divisions of the same company. It is clear that this represents a very small proportion of the world's software companies and company in-house developers. In addition, there is evidence that the majority of small and very small software organizations are not adopting standards such as CMMI. For example, an Australian study [8] found that small organizations considered that adopting CMMI "would be infeasible".

Further investigation of the SEI CMMI appraisal data reveals that in the case of Ireland - a country whose indigenous software industry is primarily made of small to medium sized organizations (SME) - fewer than 10 CMMI appraisals were conducted during the ten year period 2001 - 2011, from a population of more than 900 software companies. Therefore it is also clear that the Irish software industry is largely ignoring the most highly-publicized SPI models. In the case of CMMI (and its predecessor CMM), Staples and Niazi [9] discovered, after systematically reviewing 600 papers, that there has been little published evidence about those organizations who have decided not to adopt CMMI.

Though it is not new to claim that SPI has an associated cost, many companies are deterred from investigating SPI models because of a perceived cost. Managers' perceptions are that SPI means increased documentation and bureaucracy [3]. Such a perception is widespread and is seen as a 'feature' of standards such as CMMI. Whether or not this is true is a debatable point. The fact that managers associate CMMI with increased overhead means that most small companies do not see the model as being a viable solution or even worthy of investigation. 
There is evidence [2, 3, 4] that the majority of small and very small software organizations are not adopting existing standards / proven best practice models because they perceive the standards as being developed by large organizations and orientated towards large organizations, thus provoking the debate the in terms of number of employees, size does actually matter. Studies have shown that small firms' negative perceptions of process model standards are primarily driven by negative views of cost, documentation and bureaucracy. In addition, it has been reported that SMEs find it difficult to relate standards to their business needs and to justify the application of the international standards in their operations. Most SMEs cannot afford the resources for, or see a net benefit in, establishing software processes as defined by current standards (e.g. ISO/IEC 12207) and maturity models (e.g CMMI).

\subsection{Very Small Entities}

The definition of "Small" and "Very Small" Entities is challengingly ambiguous, as there is no commonly accepted definition of the terms. For example, the participants of the 1995 Capability Maturity Model (CMM) tailoring workshop [10] could not even agree on what "small" really meant. Subsequently in 1998 SEPG conference panel on the CMM and small projects small was defined as "3-4 months in duration with 5 or fewer staff". Johnson and Brodman [11] define a small organization as "fewer than 50 software developers and a small project as fewer than 20 software developers".

To take a legalistic perspective the European Commission [12] defines three levels of small to medium-sized enterprise (SME) as being: Small to medium - "employ fewer than 250 persons and which have an annual turnover not exceeding 50 million Euro, and/or an annual balance sheet total not exceeding 43 million Euro"; Small - "which employ fewer than 50 persons, and whose annual turnover and/or annual balance sheet total does not exceed 10 million Euro" and Micro - "which employ fewer than 10 persons and whose annual turnover does not exceed 2 million euro".

To better understand the dichotomy between the definitions above it is necessary to examine the size of software companies operating in the market today. In Europe, for instance, $85 \%$ of the Information Technology (IT) sector's companies have 1 to 10 employees. In the context of indigenous Irish software firms 1.9\% (10 companies), out of a total of 630 employed more than 100 people whilst $61 \%$ of the total employed 10 or fewer, with the average size of indigenous Irish software firms being about 16 employees [4]. In Canada, the Montreal area was surveyed, it was found that $78 \%$ of software development enterprises have less than 25 employees and $50 \%$ have fewer than 10 employees [2]. In Brazil, small IT companies (less than 50 employees) represent about $70 \%$ of the total number of companies [13].

The term "very small entity" had been defined by the ISO/IEC JTC1/SC7 Working Group (WG) 24 and subsequently adopted for use in the new ISO/IEC 29110 software process lifecycle standard as being "an entity (enterprise, organization, department or project) having up to 25 people" [2]. 


\subsection{VSE and standards}

In a time when software quality is a key to competitive advantage, the use of ISO/IEC systems and software engineering standards remains limited to a few of the most popular ones, such as ISO 9000 . Research shows that VSEs can find it difficult to relate ISO/IEC standards to their business needs and to justify the application of the standards to their business practices. Most of these VSEs can't afford the resources in number of employees, expertise, cost, and time - or see a net benefit in establishing software life-cycle processes. There is sometimes a disconnect between the short-term vision of the organization, looking at what will keep it in business for another six months or so, and the long-term benefits of gradually improving the ways the company can manage its software development and maintenance. A primary reason cited by many small software organizations for this lack of adoption of such ISO standards, is the perception that they have been developed by and for large multinational software companies and not with the small organisation in mind [4]. Subsequently, VSEs have no or very limited ways to be recognized as enterprises that produce quality software systems in their domain and may therefore be cut off from some economic activities.

Small software organizations, in the first instance, focus exclusively on survival. This, in part, explains the success of agile methodologies whose 'light', nonbureaucratic techniques support companies in survival mode attempting to establish good, fundamental software development practices. Though CMMI is firmly anchored in the belief that better processes mean better products, many small Irish software product companies are merely concerned about getting a product released to the market as quickly as possible. Development models, such as those within the agile approach, rather than CMMI or ISO 9000, are perceived as supporting this objective. This clearly poses questions for CMMI and ISO 9000 researchers. However, if SPI models are to be more widely deployed by early stage (start-ups) companies, existing models may have to be broadened to take account of the necessity for these companies to meet their development targets and 'walk before they can run' [4]

\section{The ISO/IEC 29110 Standard}

Accordingly there is a need to help such organizations understand and use the concepts, processes and practices proposed in the ISO/IEC JTC1/SC7's international software engineering standards. The ISO/IEC 29110 standard "Lifecycle profiles for Very Small Entities" [1] is aimed at addressing the issues identified above and addresses the specific needs of VSEs [2]. The approach [2] used to develop ISO/IEC 29110 started with the pre-existing international standard ISO/IEC 12207 [14] dedicated to software process lifecycles. The overall approach consisted of three steps: (1) Selecting ISO/IEC 12207 process subset applicable to VSEs of up to 25 employees; (2) Tailor the subset to fit VSE needs; and (3) Develop guidelines for VSEs.

The core characteristic of the entities targeted by ISO/IEC 29110 is size, however there are other aspects and characteristics of VSEs that may affect profile preparation 
or selection, such as: Business Models (commercial, contracting, in-house development, etc.); Situational factors (such as criticality, uncertainty environment, etc.); and Risk Levels. Creating one profile for each possible combination of values of the various dimensions introduced above would result in an unmanageable set of profiles. Accordingly VSE's profiles are grouped in such a way as to be applicable to more than one category. Table 1 illustrates a Profile Group which contains three profiles (labeled A, B and C) that are mapped to nine combinations of business models and situational factors.

Table 1. Allocating VSE characteristics to profile groups

\begin{tabular}{|l|l|l|l|}
\hline & \multicolumn{3}{|c|}{ Profile Situational Factors } \\
\hline $\begin{array}{l}\text { Business } \\
\text { Models }\end{array}$ & Critical & $\begin{array}{l}\text { User } \\
\text { Uncertainty }\end{array}$ & $\begin{array}{l}\text { Environment } \\
\text { Change }\end{array}$ \\
\hline Contract & Profile A & Profile A & Profile A \\
\hline In-House & Profile $C$ & Profile B & Profile A \\
\hline Commercial & Profile $B$ & Profile A & Profile A \\
\hline
\end{tabular}

Profile Groups are a collection of profiles which are related either by composition of processes (i.e. activities, tasks), or by capability level, or both. The "Generic" profile group has been defined [15] as applicable to a vast majority of VSEs that do not develop critical software and have typical situational factors. This profile group does not imply any specific application domain, however, it is envisaged that in the future new domain-specific sub-profiles may be developed in the future. Table 2 illustrates this profile group as a collection of four profiles, providing a progressive approach to satisfying the requirements of profile group.

Table 2. Graduated profile of the Generic profile group

\begin{tabular}{|l|l|l|l|}
\hline & \multicolumn{3}{|c|}{ Generic Profile Group } \\
\hline Entry & Basic & Intermediate & Advanced \\
\hline & & & \\
\hline & & & \\
\hline & & & \\
\hline & & & \\
\hline
\end{tabular}

To date the Basic Profile [1] has been pupblished, the purpose of which is to define a software development and project management guide for a subset of processes and outcomes appropriate for characteristics and needs of VSEs.

\subsection{Engineering and management guide}

At the core of this standard is a Management and Engineering Guide (ISO/IEC 29110-5) [1] focusing on Project Management and Software Implementation as 
illustrated in figure 1. The purpose of the Project Management process is to establish and carry out in a systematic way the tasks of a software implementation project, which complies with the project's objectives in terms of quality, time and cost. Project Management generates a Project Plan to direct the software project. During the execution of the project Change Requests may cause revisions to the Project Plan. The project is the subject of Project Assessment and Control during the lifetimes of the project until the Software Implementation is complete and Project Closure occurs. Software Implementation (SI) produces a specified software system implemented as a software product or service. This process starts with the establishment of Software Requirements, after which Architectural and Detailed Design are produced. Software is the Constructed and verified using Integration and Test procedures. The final staged being product delivery to the customer.
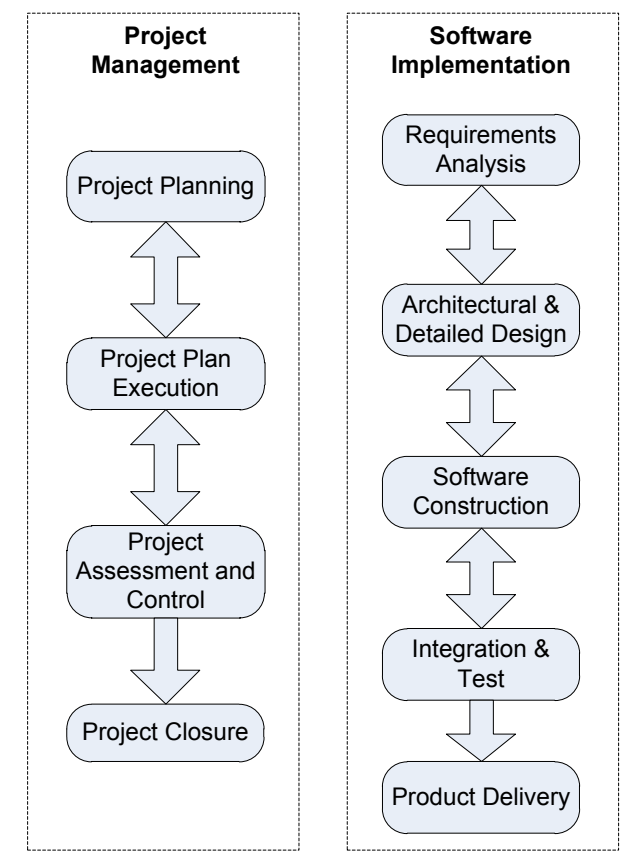

Fig 1. ISO/IEC 29110 Process Diagrams

\section{Deployment assistance}

In order to assist with the deployment of ISO/IEC 29110 and to provide guidance on the actual implementation of ISO/IEC 29110-5 in VSEs a series of Deployment Packages and Implementation Guides have been developed to define guidelines and explain in more detail the processes defined in the ISO/IEC 29110 profiles.

The issues of assistance to VSEs in understanding and adopting standards, as outlined above, must be addressed. To this end, some members of the ISO/IEC 
JTC1/SC7 WG 24 have produced a set of "Deployment Packages" (DP) which are freely available from [16]. A DP is a set of artifacts developed to facilitate the implementation of a set of practices, of the selected framework, in a VSE. A DP is not a process reference model (i.e. it is not prescriptive). The elements of a typical DP are: description of processes, activities, tasks, roles and products, template, checklist, example, reference and mapping to standards and models, and a list of tools. The mapping is only given as information to show that a deployment package has explicit links to standards, such as ISO/IEC 12207, or models, such as the CMMI for Development, hence by deploying and implementing the package, a VSE can see its concrete step to achieve or demonstrate coverage. Packages are designed such that a VSE can implement its content, without having to implement the complete framework at the same time. The table of content of a deployment package is illustrated in figure 2.

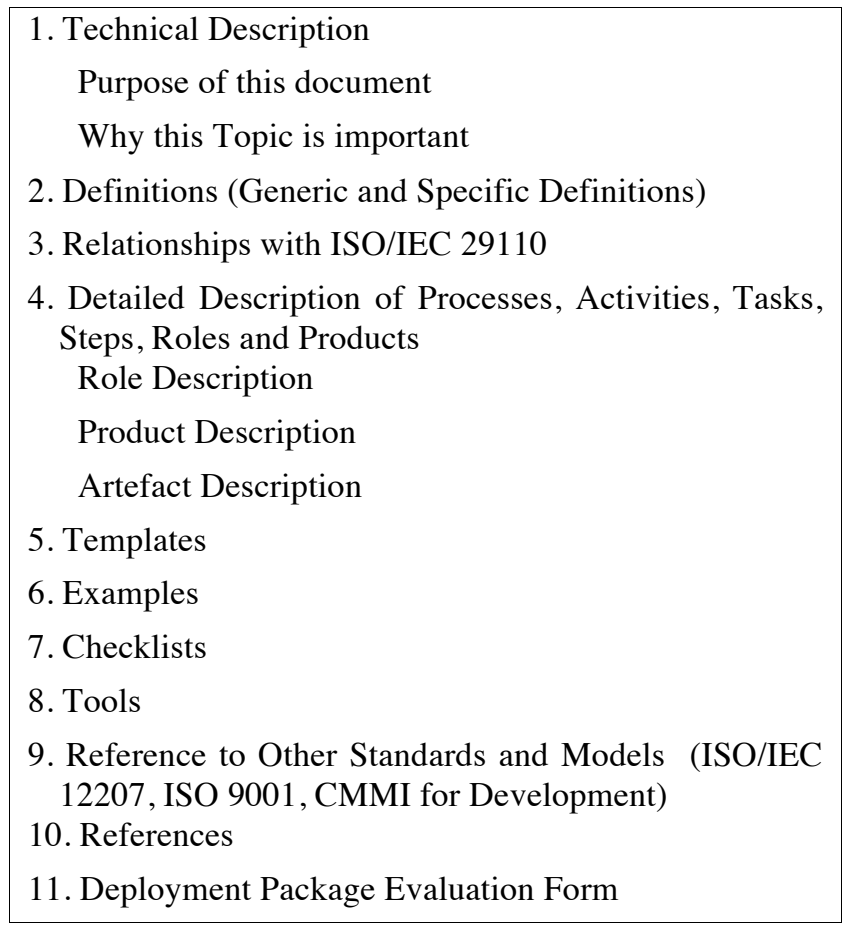

Fig. 2. Table of Content of a deployment package.

In addition a series of Implementation Guides have been developed to help implement a specific process supported by a tool and are freely available from [16]. To date a small number of implementation guides have been developed. These include:

- Version Control with CVS

- Version Control with SVN

- $\quad$ Project Management with GForge

- Issue tracking with GForge

- Software Process Improvement with OpenOffice Calc. 


\section{Pilot projects}

The working group (ISO/IEC JTC1/SC7 WG 24) behind the development of this standard is advocating the use of pilot projects as a mean to accelerate the adoption and utilization of ISO/IEC 29110 by VSEs around the world. Pilot projects are an important mean of reducing risks and learning more about the organizational and technical issues associated with the deployment of new software engineering practices. A successful pilot project is also an effective means of building adoption of new practices by members of a VSE. Pilot projects are based on the ISO/IEC 29110-5 Management and engineering guide [1] and the deployment package(s). In particular these are aimed to collect, as a minimum, the following data:

- Effort and time to deploy by the VSE

- Usefulness for the VSE

- Verification of the understanding of the VSE

- Self-assessments data - A self-assessment at the beginning of the pilot and at the end of the pilot project DP

To date a series of pilot projects have been completed in several countries utilizing some of the deployment packages developed. For example in Canada a pilot study has been conducted with an IT department with a staff of 4: 1 analyst and 3 developers, who were involved in the translation and implemented 3 DPs: Software Requirements, Version Control, Project Management. In Belgium a VSE of 25 people started with a process assessment phase aiming to identify strengths and weaknesses in development related processes. This company is now working on improvement actions mainly based on the following Deployment Packages: Requirement Analysis, Version Control, and Project Management. In France, a pilot study [17] was conducted with a 14-people VSE that builds and sells counting systems about the frequenting of natural spaces and public sites. In addition a further series of pilot projects are currently underway in Canada, Ireland, Belgium and France, with further pilot projects planned in the near future.

\subsection{Trials to date}

To date we have published [17] the final conclusions and results of one pilot project that conducted with a 14-person VSE based in France, which successfully implemented ISO/IEC 29110 processes practices utilising the available Deployment Packages. From which we have identified some potential additional infrastructure and support process activities and suggestions for future evolution of ISO/IEC 29110 Process Profiles. A further series of pilot projects are currently underway in research laboratories and enterprises in Canada, Ireland, Belgium and France, with further pilot projects planned in the near future. 


\section{Discussion}

As ISO/IEC 29110 is an emerging standard there is much work yet to be completed. The main remaining work item is to finalize the development of the remaining three profiles: (a) Entry - a six person-months effort project or a start-up VSEs; (b) Intermediate - Management of more than one project and (c) Advanced business management and portfolio management practices. In addition the development of additional Profile Groups for other domains such as critical software, game industry, scientific software development are being studied

With any new initiative there is much to be learnt from conducting pilot projects. One issue of major importance to VSEs which is emerging from these pilot projects and similar work by the ISO working group is the need for a light-weight flexible approach to process assessment. Whilst work is currently underway on an assessment mechanism for ISO/IEC 29110 [18], a clear niche market need is emerging which may force the process assessment community to change their views on how process assessments are carried out for VSEs. In particular there is a strong need to ensure that VSEs are not required to invest the anything similar in terms of time, money and other resources on process assessments, as may be expected from their larger SMEs (small and medium enterprises), or even MNC (multinational corporations) counterparts. Indeed some form of self-assessment, possibly supported by Internet based tools, along with periodic spot-checks may be suitable alternative to meet the unique needs of VSEs. It is clear that the process assessment community will have to rethink process assessment, new methods and ideas for assessing processes in VSEs.

Acknowledgments: This work is supported, in part, by Science Foundation Ireland grant 03/CE2/I303_1 to Lero, the Irish Software Engineering Research Centre (www.lero.ie).

\section{References}

1. International Organization for Standardization (ISO): ISO/IEC TR 29110-5-1-2 Software Engineering - Lifecycle Profiles for Very Small Entities (VSEs) Part 5-1-2: Management and engineering guide: Generic profile group: Basic profile, Geneva (2011)

2. Laporte, C.Y., Alexandre, S., O'Connor, R.: A Software Engineering Lifecycle Standard for Very Small Enterprises. In: O'Connor, R., et al. (eds.) Proceedings of EuroSPI. CCIS, vol. 16, pp. 129-141. Springer, Heidelberg (2008)

3. Coleman, G., O'Connor, R.: Investigating Software Process in Practice: A Grounded Theory Perspective. Journal of Systems and Software 81 (5), 772-784 (2008)

4. O'Connor R. and Coleman G., Ignoring 'Best Practice': Why Irish Software SMEs are rejecting CMMI and ISO 9000, Australasian Journal of Information Systems, Vol. 16, No. $1,2009$.

5. Ahern, D.M., Clouse, A., Turner, R., 2004. CMMI Distilled: A Practical Introduction to Integrated Process Improvement, 2nd Ed, Addison Wesley, Boston

6. ISO 9000:2005, Quality management system. International Organization for Standardization: Geneva, Switzerland (2005).

7. CMMI Published Appraisal Results List, Software Engineering Institute, http://sas.sei.cmu.edu/pars/ 
8. Staples, M, Niazi M, Jeffery, R, Abrahams, A, Byatt, P and Murphy, R, 2007, An exploratory study of why organizations do not adopt CMMI, The Journal of Systems and Software, Vol. 80, No. 6, 883-895

9. Staples, M., Niazi, M., 2006. Systematic Review of Organizational Motivations for Adopting CMM-based SPI. Technical Report PA005957, National ICT Australia.

10. Ginsberg, M., Quinn, L.: Process Tailoring and the Software Capability Maturity Model, Software Engineering Institute, CMU/SEI-94-TR-024 (November 1995)

11. Johnson, D., Brodman, J.: Applying the CMM to Small Organizations and Small Projects. In: Proceedings of Software Engineering Process Group Conference, Chicago (1998)

12. European Commission, The New SME Definition: User Guide and Model Declaration (2005),

http://europa.eu.int/comm/enterprise/enterprise_policy/sme_definition/sme_user_guide.pdf

13. Anacleto, A., von Wangenheim, C.G., Salviano, C.F., Savi, R.: Experiences gained from applying ISO/IEC 15504 to small software companies in Brazil. In: 4th International SPICE Conference on Process Assessment and Improvement, Lisbon, Portugal (April 2004)

14. ISO/IEC 12207:2008, Information technology - Software life cycle processes. International Organization for Standardization/International Electrotechnical Commission: Geneva, Switzerland (2008).

15. O'Connor R. and Laporte.C.Y., Towards the provision of assistance for very small entities in deploying software lifecycle standards. In Proceedings of the 11th International Conference on Product Focused Software (PROFES '10). ACM, (2010)

16. ISO/IEC JCT1/SC7 Working Group 24 Deployment Packages repository, http://profs.logti.etsmtl.ca/claporte/English/VSE/index.html

17. Ribaud, V., Saliou, P., O'Connor, R., Laporte, C.: Software Engineering Support Activities for Very Small Entities. In: Riel, et al. (eds.) Systems, Software and Services Process Improvement. CCIS, vol. 99, pp. 165-176. Springer-Verlag, Heidelberg (2010)

18 International Organization for Standardization (ISO). ISO/IEC TR 29110-3, Software Engineering - Lifecycle Profiles for Very Small Entities (VSE) - Part 3: Assessment Guide. Geneva (2011)

19. O'Connor, R., Laporte, C.: Deploying Lifecycle profiles for Very Small Entities: An Early Stage Industry View. In: O'Connor, et al. (eds.) Software Process Improvement and Capability Determination. CCIS, vol. 155, pp. 227-230. Springer-Verlag, Heidelberg (2011) 